PROCEEDINGS OF THE

AMERICAN MATHEMATICAL SOCIETY

Volume 141, Number 12, December 2013, Pages 4241-4247

S 0002-9939(2013)11701-6

Article electronically published on August 5, 2013

\title{
INVERTIBLE WEIGHTED SHIFT OPERATORS WHICH ARE $m$-ISOMETRIES
}

\author{
MUNEO CHŌ, SCHÔICHI ÔTA, AND KÔTARÔ TANAHASHI \\ (Communicated by Richard Rochberg)
}

\begin{abstract}
For a bounded linear operator $T$ on a complex Hilbert space $\mathcal{H}$, let $\Delta_{T, m}=\sum_{k=0}^{m}(-1)^{k}\left(\begin{array}{c}m \\ k\end{array}\right) T^{* m-k} T^{m-k}$ for $m \in \mathbb{N} . T$ is called an $m$ isometry if $\Delta_{T, m}=0$. In this paper, for every even number $m$, we give an example of invertible $(m+1)$-isometry which is not an $m$-isometry. Next we show that if $T$ is an $m$-isometry, then the operator $\Delta_{T, m-1}$ is not invertible.
\end{abstract}

\section{INTRODUCTION}

J. Agler and M. Stankus published excellent papers about $m$-isometric operators, 1], 2 and 3. They showed that $m$-isometries have interesting spectral properties. For example, if $T$ is an $m$-isometry, then the approximate point spectrum of $T$ lies on the unit circle. In [10, Patel showed that Weyl's theorem holds for a 2-isometry. Applying Uchiyama and Tanahashi's result [1], in [8] we showed that if $T$ is an $m$-isometry, then $T$ has the single valued extension property. Let $\mathcal{H}$ be a complex Hilbert space and $B(\mathcal{H})$ be a set of all bounded linear operators on $\mathcal{H}$. Let $\left(\begin{array}{c}m \\ k\end{array}\right)$ be the binomial coefficient. For an operator $T \in B(\mathcal{H})$, let

$$
\Delta_{T, m}=\sum_{k=0}^{m}(-1)^{k}\left(\begin{array}{c}
m \\
k
\end{array}\right) T^{* m-k} T^{m-k} .
$$

$T$ is said to be an $m$-isometry if $\Delta_{T, m}=0$. Agler and Stankus proved that if $T$ is an $m$-isometry, then $\Delta_{T, m-1} \geq 0$ (Proposition 1.5, [1]). It is easy to see that $T^{*} \Delta_{T, m} T-\Delta_{T, m}=\Delta_{T, m+1}$. Hence it holds that if $T$ is an $m$-isometry, then $T$ is an $(m+1)$-isometry. In [7], T. Bermudez, A. Martinon and E. Negrin studied characterizations of weighted shift operators which are $m$-isometries. In [1], Agler and Stankus proved that if $m$ is even and $T$ is an invertible $m$-isometry, then $T$ is an $(m-1)$-isometry. In [4] A. Athavale proved that if $S$ is a unilateral weighted shift

Received by the editors October 2, 2011 and, in revised form, January 31, 2012.

2010 Mathematics Subject Classification. Primary 47B37.

Key words and phrases. Hilbert space, $m$-isometry, bilateral weighted shift.

The first author's research was partially supported by Grant-in-Aid for Scientific Research, No. 20540192.

The second author's research was partially supported by Grant-in-Aid for Scientific Research, No. 20540178.

The third author's research was partially supported by Grant-in-Aid for Scientific Research, No. 20540184.

(C)2013 American Mathematical Society Reverts to public domain 28 years from publication 
$S e_{n}=w_{n} e_{n+1}$ with weights $w_{n}=\sqrt{1+\frac{m}{n}}$ for $n=1,2,3, \cdots$, then $S$ is an $(m+1)$ isometry which is not an $m$-isometry. The operator $S$ is not invertible. We have not seen invertible $(m+1)$-isometries which are weighted shifts for $m$ even. We give an example of an invertible $(m+1)$-isometry which is not an $m$-isometry for every even number $m$. Next we prove that power bounded $m$-isometries are isometries, and if $T$ is an $m$-isometry for a natural number $m \geq 2$, then the operator $\Delta_{T, m-1}$ is not invertible.

\section{INVERTIBLE WEIGHTED SHIFT OF AN $m$-ISOMETRY}

Agler and Stankus showed the following result.

Proposition A (Proposition 1.23, [1]). If $T$ is an invertible $m$-isometry and $m$ is even, then $T$ is an $(m-1)$-isometry.

Their proof is fine. We give another proof.

Proof. Since $T$ is an $m$-isometry, it holds that

$$
\Delta_{T, m-1}=\sum_{k=0}^{m-1}(-1)^{k}\left(\begin{array}{c}
m-1 \\
k
\end{array}\right) T^{* m-1-k} T^{m-1-k} \geq 0 .
$$

It is easy to see that $T^{-1}$ also is an $m$-isometry. Since $T^{-1}$ is an $m$-isometry, it holds that

$$
\Delta_{T^{-1}, m-1}=\sum_{k=0}^{m-1}(-1)^{k}\left(\begin{array}{c}
m-1 \\
k
\end{array}\right)\left(T^{-1}\right)^{* m-1-k}\left(T^{-1}\right)^{m-1-k} \geq 0 .
$$

Since $m-1$ is an odd number, by (2.2) we have

$$
\begin{aligned}
-\Delta_{T, m-1} & =\sum_{k=0}^{m-1}(-1)^{k}\left(\begin{array}{c}
m-1 \\
k
\end{array}\right) T^{* k} T^{k} \\
& =\sum_{k=0}^{m-1}(-1)^{k}\left(\begin{array}{c}
m-1 \\
k
\end{array}\right) T^{* m-1}\left(T^{-1}\right)^{* m-1-k}\left(T^{-1}\right)^{m-1-k} T^{m-1} \\
& =T^{* m-1}\left(\Delta_{T^{-1}, m-1}\right) T^{m-1} \geq 0 .
\end{aligned}
$$

Hence $\Delta_{T, m-1} \leq 0$. By $(2.1)$ we have $\Delta_{T, m-1}=0$, so the proof is complete.

For every even number $m$, we give an example of an invertible $(m+1)$-isometry $T$ which is not an $m$-isometry.

Theorem 1. For any even number $m$, there exists an invertible $(m+1)$-isometry $T$ which is not an m-isometry.

Proof. Let $m$ be any even number and put $\psi(x)=x(x+1) \cdots(x+m-1)$. Then $\psi(x)$ is a polynomial of even degree $m$. Hence $\psi(n) \geq 0$ for $n=0, \pm 1, \pm 2, \cdots$. So, for any $k>0$, we have $\psi(n)+k>0$ for $n=0, \pm 1, \pm 2, \cdots$. Let

$$
w_{n}=\sqrt{\frac{\psi(n+1)+k}{\psi(n)+k}}>0 \text { for } n=0, \pm 1, \pm 2, \cdots
$$


Let $\left\{e_{n}\right\}_{n=-\infty}^{\infty}$ be an orthonormal basis of $\mathcal{H}=\ell^{2}$ and $T$ be a bilateral weighted shift such that $T e_{n}=w_{n} e_{n+1}$. We define

$$
\begin{aligned}
& I_{m, n}=w_{n}^{2} w_{n+1}^{2} \cdots w_{n+m-1}^{2}-\left(\begin{array}{c}
m \\
1
\end{array}\right) w_{n}^{2} w_{n+1}^{2} \cdots w_{n+m-2}^{2} \\
& +\left(\begin{array}{c}
m \\
2
\end{array}\right) w_{n}^{2} w_{n+1}^{2} \cdots w_{n+m-3}^{2}+\cdots+(-1)^{m-1}\left(\begin{array}{c}
m \\
m-1
\end{array}\right) w_{n}^{2}+(-1)^{m} .
\end{aligned}
$$

Note that $T$ is an $m$-isometry if and only if $I_{m, n}=0$ for $n=0, \pm 1, \pm 2, \cdots$. To show $T$ is an $(m+1)$-isometry, we will show that $I_{m+1, n}=0$ for $n=0, \pm 1, \pm 2, \cdots$. We define a function $f(x)$ as

$$
\begin{aligned}
f(x)= & x^{n+m-1}(1-x)^{m+1} \\
= & x^{n+m-1}-\left(\begin{array}{c}
m+1 \\
1
\end{array}\right) x^{n+m}+\left(\begin{array}{c}
m+1 \\
2
\end{array}\right) x^{n+m+1} \\
& +\cdots+(-1)^{m}\left(\begin{array}{c}
m+1 \\
m
\end{array}\right) x^{n+2 m-1}+(-1)^{m+1} x^{n+2 m} .
\end{aligned}
$$

Note that $f^{(m)}(1)=0$. By differentiating $m$ times, we have

$$
\begin{aligned}
0= & f^{(m)}(1) \\
= & (n+m-1)(n+m-2) \cdots n \\
& -\left(\begin{array}{c}
m+1 \\
1
\end{array}\right)(n+m)(n+m-1) \cdots(n+1) \\
& +\cdots+(-1)^{m}\left(\begin{array}{c}
m+1 \\
m
\end{array}\right)(n+2 m-1)(n+2 m-2) \cdots(n+m) \\
& +(-1)^{m+1}(n+2 m)(n+2 m-1) \cdots(n+m+1) .
\end{aligned}
$$

Hence

$$
\begin{aligned}
&(-1)^{m+1} I_{m+1, n}(\psi(n)+k) \\
&=(n+m+1)(n+m+2) \cdots(n+2 m)+k \\
&-\left(\begin{array}{c}
m+1 \\
1
\end{array}\right)\{(n+m)(n+m+1) \cdots(n+2 m-1)+k\} \\
&+\cdots+(-1)^{m}\left(\begin{array}{c}
m+1 \\
m
\end{array}\right)\{(n+1)(n+2) \cdots(n+m)+k\} \\
&+(-1)^{m+1}\{n(n+1)(n+2) \cdots(n+m-1)+k\} \\
&= f^{(m)}(1)+k(1-1)^{m+1}=0 .
\end{aligned}
$$

This implies that $I_{m+1, n}=0$. Hence $T$ is an $(m+1)$-isometry.

To show that $T$ is not an $m$-isometry, we will show that $I_{m, n} \neq 0$ for $n=$ $0, \pm 1, \pm 2, \cdots$. We define a function $g(x)$ as

$$
\begin{aligned}
g(x)= & x^{n+m-1}(1-x)^{m} \\
= & x^{n+m-1}-\left(\begin{array}{c}
m \\
1
\end{array}\right) x^{n+m}+\left(\begin{array}{c}
m \\
2
\end{array}\right) x^{n+m+1} \\
& +\cdots+(-1)^{m-1}\left(\begin{array}{c}
m \\
m-1
\end{array}\right) x^{n+2 m-2}+(-1)^{m} x^{n+2 m-1} .
\end{aligned}
$$


Note that $g^{(m)}(1)=m !(-1)^{m}$. By differentiating $m$ times, we have

$$
\begin{aligned}
m !(-1)^{m}= & g^{(m)}(1) \\
= & (n+m-1)(n+m-2) \cdots n \\
& -\left(\begin{array}{c}
m \\
1
\end{array}\right)(n+m)(n+m-1) \cdots(n+1) \\
& +\cdots+(-1)^{m-1}\left(\begin{array}{c}
m \\
m-1
\end{array}\right)(n+2 m-2)(n+2 m-3) \cdots(n+m-1) \\
& +(-1)^{m}(n+2 m-1)(n+2 m-2) \cdots(n+m) .
\end{aligned}
$$

Hence

$$
\begin{aligned}
&(-1)^{m} I_{m, n}(\psi(n)+k) \\
&=(n+m)(n+m+1) \cdots(n+2 m-1)+k \\
&-\left(\begin{array}{c}
m \\
1
\end{array}\right)\{(n+m-1)(n+m) \cdots(n+2 m-2)+k\} \\
&+\cdots+(-1)^{m-1}\left(\begin{array}{c}
m \\
m-1
\end{array}\right)\{(n+1)(n+2) \cdots(n+m)+k\} \\
&+(-1)^{m}\{n(n+1)(n+2) \cdots(n+m-1)+k\} \\
&= g^{(m)}(1)+k(1-1)^{m}=m !(-1)^{m} .
\end{aligned}
$$

This implies that $I_{m, n}=m !(\psi(n)+k)^{-1} \neq 0$. Hence $T$ is not an $m$-isometry.

Finally, since $w_{n} \rightarrow 1(n \rightarrow \pm \infty)$, we have $\sigma(T)=\{\lambda:|\lambda|=1\}$ by Proposition 2.6.8 (b) of [9]. Therefore $T$ is invertible. The proof is complete.

Remark 1. This result provides an example of an $(m+1)$-isometry $T$ which is not an $m$-isometry if we take $T e_{n}=w_{n} e_{n+1}$ for $n=1,2, \cdots$ where

$$
w_{n}=\sqrt{\frac{(n+1)(n+2) \cdots(n+m)+k}{n(n+1) \cdots(n+m-1)+k}}
$$

with $0 \leq k$. If $k=0$, then

$$
w_{n}=\sqrt{\frac{(n+1)(n+2) \cdots(n+m)+0}{n(n+1) \cdots(n+m-1)+0}}=\sqrt{\frac{n+m}{n}} .
$$

This is the result of A. Athavale, Proposition 8 of [4].

\section{Some Properties of $m$-ISOMETRIES}

An operator $T \in B(\mathcal{H})$ is said to be power bounded if there exists a positive number $M$ such that $\left\|T^{n}\right\| \leq M$ for every $n \in \mathbb{N}$.

Theorem 2. A power bounded m-isometry is an isometry.

To prove this result, we will use Berberian's method (cf. [6], [12]). 
Proposition (Lemma 2.7, [12]). Let $\mathcal{H}$ be a complex Hilbert space. Then there exist a Hilbert space $\mathcal{H}^{\circ} \supset \mathcal{H}$ and a unital linear map $\circ: B(\mathcal{H}) \rightarrow B\left(\mathcal{H}^{\circ}\right)$ such that

$$
\begin{aligned}
& \text { (i) }(S T)^{\circ}=S^{\circ} T^{\circ},\left(T^{*}\right)^{\circ}=\left(T^{\circ}\right)^{*},\|T\|=\left\|T^{\circ}\right\|, \\
& \text { (ii) } S \leq T \Longrightarrow S^{\circ} \leq T^{\circ}, \\
& \text { (iii) } \sigma(T)=\sigma\left(T^{\circ}\right), \sigma_{a}(T)=\sigma_{a}\left(T^{\circ}\right)=\sigma_{p}\left(T^{\circ}\right) .
\end{aligned}
$$

We prepare notation and a lemma. For a unit vector $x \in \mathcal{H}$, assume that $\left(T^{*} T-I\right) x=a_{1} x$. Let $a_{n}=\left\langle\Delta_{T, n} x, x\right\rangle$ and $b_{n}=\left\|T^{n} x\right\|^{2}$ for $n=1,2,3, \cdots$. Then we have the following lemma.

Lemma. For an operator $T \in B(\mathcal{H})$ with the above notation, it holds that

$$
b_{n}=a_{n}+\left(\begin{array}{c}
n \\
n-1
\end{array}\right) a_{n-1}+\left(\begin{array}{c}
n \\
n-2
\end{array}\right) a_{n-2}+\cdots+n a_{1}+1 .
$$

Proof. Note that

$$
b_{1}=\|T x\|^{2}=\left\langle T^{*} T x, x\right\rangle=\left\langle a_{1} x+x, x\right\rangle=a_{1}+1 .
$$

Since

$$
\begin{aligned}
T^{* 2} T^{2} & =\left(T^{* 2} T^{2}-2 T^{*} T+I\right)+2\left(T^{*} T-I\right)+I \\
& =\Delta_{T, 2}+2 \Delta_{T, 1}+I
\end{aligned}
$$

we have

$$
\begin{aligned}
b_{2} & =\left\langle T^{* 2} T^{2} x, x\right\rangle \\
& =\left\langle\Delta_{T, 2} x, x\right\rangle+2\left\langle\Delta_{T, 1} x, x\right\rangle+\langle x, x\rangle \\
& =a_{2}+2 a_{1}+1 .
\end{aligned}
$$

Hence (3.1) holds for $n=1,2$. Note that

$$
\begin{aligned}
t^{n} & =(t-1+1)^{n} \\
& =(t-1)^{n}+\left(\begin{array}{c}
n \\
1
\end{array}\right)(t-1)^{n-1}+\left(\begin{array}{c}
n \\
2
\end{array}\right)(t-1)^{n-2}+\cdots+n(t-1)+1 .
\end{aligned}
$$

Hence

$$
T^{* n} T^{n}=\Delta_{T, n}+\left(\begin{array}{c}
n \\
1
\end{array}\right) \Delta_{T, n-1}+\left(\begin{array}{c}
n \\
2
\end{array}\right) \Delta_{T, n-2}+\cdots+n \Delta_{T, n-1}+I
$$

and

$$
\begin{aligned}
b_{n} & =\left\langle T^{* n} T^{n} x, x\right\rangle \\
& =a_{n}+\left(\begin{array}{c}
n \\
1
\end{array}\right) a_{n-1}+\left(\begin{array}{c}
n \\
2
\end{array}\right) a_{n-2}+\cdots+n a_{1}+1 \\
& =a_{n}+\left(\begin{array}{c}
n \\
n-1
\end{array}\right) a_{n-1}+\left(\begin{array}{c}
n \\
n-2
\end{array}\right) a_{n-2}+\cdots+n a_{1}+1 .
\end{aligned}
$$

This completes the proof.

Proof of Theorem 2. Let $T$ be a power bounded $m$-isometry. Since $T^{*} T-I$ is self-adjoint, it suffices to show that

$$
\sigma\left(T^{*} T-I\right)=\sigma_{a}\left(T^{*} T-I\right)=\{0\} .
$$

Assume that there exists a non-zero real number $a \in \sigma\left(T^{*} T-I\right)$. Since $a$ belongs to the approximate point spectrum of $T^{*} T-I$, by Berberian's method we consider 
an extension $\mathcal{H}^{\circ}$ of $\mathcal{H}$ and the mapping $S \rightarrow S^{\circ}$ of $B(\mathcal{H})$ into $B\left(\mathcal{H}^{\circ}\right)$. Then $a$ is an eigen-value of $T^{* \circ} T^{\circ}-I^{\circ}$ and $T^{\circ}$ is also a power bounded $m$-isometry. For simplification, we denote $T^{\circ}$ by $T$. Since $a$ is an eigen-value of $T^{*} T-I$, there exists a unit vector $x$ such that $\left(T^{*} T-I\right) x=a x$. Hence

$$
\left\langle\left(T^{*} T-I\right) x, x\right\rangle=\langle a x, x\rangle=a .
$$

Let $a_{1}=a$ and $a_{n}=\left\langle\Delta_{T, n} x, x\right\rangle$. Then since $T$ is an $m$-isometry, we have $a_{m}=$ $a_{m+1}=\cdots=a_{n}=0$ for $n>m$. By the Lemma, we have

$$
\left\|T^{n} x\right\|^{2}=\left(\begin{array}{c}
n \\
m-1
\end{array}\right) a_{m-1}+\left(\begin{array}{c}
n \\
m-2
\end{array}\right) a_{m-2}+\cdots+n a_{1}+1 .
$$

Hence

$$
\frac{\left\|T^{n} x\right\|^{2}}{\left(\begin{array}{c}
n \\
m-1
\end{array}\right)}=a_{m-1}+\frac{1}{\left(\begin{array}{c}
n \\
m-1
\end{array}\right)}\left\{\left(\begin{array}{c}
n \\
m-2
\end{array}\right) a_{m-2}+\cdots+n a_{1}+1\right\} .
$$

Since $T$ is power bounded, we have $a_{m-1}=0$ by $n \rightarrow \infty$. Repeating this, we have

$$
a_{m-1}=a_{m-2}=\cdots=a_{1}=a=0 .
$$

This is a contradiction, so the proof is complete.

Patel (Corollary 2.8, 10]) proved that if $T$ is a 2-isometry, then $1 \in \sigma\left(T^{*} T\right)$. That is, if $T$ is a 2-isometry, then $0 \in \sigma\left(\Delta_{T, 1}\right)$. We now generalize this result as follows.

Theorem 3. If $T$ is an $m$-isometry for $m \geq 2$, then $0 \in \sigma\left(\Delta_{T, m-1}\right)$.

Proof. For simplification, we denote $\Delta_{T, m-1}$ by $\Delta$. Assume that $0 \notin \sigma(\Delta)$. Since $\Delta_{T, m}=T^{*} \Delta T-\Delta=0$, it holds that $T^{*} \Delta T=\Delta$. Let $S=\Delta^{1 / 2} T \Delta^{-1 / 2}$. Then

$$
S^{*} S=\left(\Delta^{-1 / 2} T^{*} \Delta^{1 / 2}\right)\left(\Delta^{1 / 2} T \Delta^{-1 / 2}\right)=I .
$$

Hence $S$ is an isometry and $T=\Delta^{-1 / 2} S \Delta^{1 / 2}$. So $T$ is similar to the isometry $S$. Since $S$ is power bounded, $T$ is an isometry by Theorem 3 . Hence $\Delta_{T, 1}=0$, and so $\Delta_{T, m-1}=\Delta=0$. This contradicts our assumption that $0 \notin \sigma(\Delta)$, so the proof is complete.

Remark 2. Since Weyl's theorem holds for an isometry, Weyl's theorem holds for a power bounded $m$-isometry by Theorem 3 (cf. [5], [10]).

Finally we show the following result. For a 2-isometry, Patel proved it (Theorem 2.1, [10]).

Theorem 4. A power of an m-isometry is again an m-isometry.

Proof. Let $T$ be an $m$-isometry, i.e.,

$$
\Delta_{T, m}=T^{* m} T^{m}-m T^{* m-1} T^{m-1}+\cdots+(-1)^{m} I=0 .
$$

We prove $T^{k}$ is also an $m$-isometry. Write

$$
\begin{aligned}
\left(t^{k}-1\right)^{m} & =(t-1)^{m}\left(t^{k-1}+t^{k-2}+\cdots+t+1\right)^{m} \\
& =\left(t^{m}-m t^{m-1}+\cdots+(-1)^{m}\right)\left(a_{m(k-1)} t^{m(k-1)}+\cdots+a_{1} t+a_{0}\right) .
\end{aligned}
$$


Hence we have

$$
\Delta_{T^{k}, m}=\sum_{j=0}^{m(k-1)} a_{j} T^{* j}\left(\Delta_{T^{k}, m}\right) T^{j}=0 .
$$

So the proof is complete.

\section{ACKNOWLEDGMENT}

The authors would like to express their sincere thanks to the referee for a careful reading and much kind advice. The proofs became very simple because of the referee's suggestions.

\section{REFERENCES}

[1] J. Agler and M. Stankus, m-Isometric transformations of Hilbert space I, Integr. Equat. Oper. Theory, 21 (1995), 387-429. MR1321694(96j:47008a)

[2] J. Agler and M. Stankus, m-Isometric transformations of Hilbert space II, Integr. Equat. Oper. Theory, 23 (1995), 1-48. MR.1346617 (96j:47008b)

[3] J. Agler and M. Stankus, m-Isometric transformations of Hilbert space III, Integr. Equat. Oper. Theory, 24 (1996), 379-421. MR1382018 (96j:47008c)

[4] A. Athavale, Some operator theoretic calculus for positive definite kernels, Proc. Amer. Math. Soc., 112(3) (1991), 701-708. MR1068114 (92j:47050)

[5] J.V. Baxley, Some general conditions implying Weyl's theorem, Rev. Roum. Math. Pures Appl., 16 (1971), 1163-1166. MR0305107 (46:4237)

[6] S.K. Berberian, Approximate proper vectors, Proc. Amer. Math. Soc., 13 (1962), 111-114. MR.0133690 (24:A3516)

[7] T. Bermudez, A. Martinon and E. Negrin, Weighted shift operators which are $m$-isometry, Integr. Equ. Oper. Theory, 68 (2010), 301-312. MR.2735438 (2012a:47074)

[8] M. Chō, S. Ôta, K. Tanahashi, and A. Uchiyama, Spectral properties of $m$-isometric operators, Funct. Anal. Approx. Comput., 4:2 (2012), 33-39.

[9] J.B. Conway, The Theory of Subnormal Operators, Math. Surveys and Monographs 36, Amer. Math. Soc., 1991. MR.1112128 (92h:47026)

[10] S.M. Patel, 2-Isometric operators, Glasnik Mat., 37 (2002), 143-147. MR.1918101 (2003f:47003)

[11] A. Uchiyama and K. Tanahashi, Bishop's property $(\beta)$ for paranormal operators, Operators and Matrices, 3 (2009), 517-524. MR2597677(2011b:47047)

[12] D. Xia, Spectral theory of hyponormal operators, Birkhäuser Verlag, Boston, 1983. MR806959 $(87 \mathrm{j}: 47036)$

Department of Mathematics, Kanagawa University, Yokohama 221-8686, Japan

E-mail address: chiyom01@kanagawa-u.ac.jp

Department of Content and Creative Design, Kyushu University, Fukuoka 815-8540, JAPAN

E-mail address: ota@design.kyushu-u.ac.jp

Department of Mathematics, Tohoku Pharmaceutical University, Sendai 981-8558, JAPAN

E-mail address: tanahasi@tohoku-pharm.ac.jp 